\title{
The exponential dielectric relaxation dynamics in a secondary alcohol's supercooled liquid and glassy states
}

\author{
O. E. Kalinovskaya and J. K. Vij ${ }^{\mathrm{a})}$ \\ Laboratory of Advanced Materials, Department of Electronic and Electrical Engineering, Trinity College, \\ University of Dublin, Dublin 2, Ireland
}

(Received 30 August 1999; accepted 5 November 1999)

\begin{abstract}
To gain insight into a recent observation that the prominent, Debye-type relaxation process observed for a primary alcohol may not be the $\alpha$-relaxation process associated with molecular diffusion of a liquid [Europhys. Lett. 40, 549 (1997), J. Chem. Phys. 107, 1086 (1997)], the dielectric spectra of an uncrystallizable secondary alcohol, 5-methyl-2-hexanol, has been investigated by broadband spectroscopy. Measurements made over a temperature range from 110 to $298 \mathrm{~K}$ showed that three relaxation processes occur. Processes I and II have a non-Arrhenius variation of the relaxation rate with temperature, and process III an Arrhenius. Only process I, the slowest of the three, has a single relaxation rate, the other two, a broad distribution. The contribution to permittivity from process II was 0.8 , i.e., $\sim 3 \%$ of the static permittivity, and from process III, the fastest was 0.1 , i.e., $\sim 0.3 \%$. It is argued that the mechanism of process $\mathrm{I}$ is the breaking followed by dipolar reorientation and reforming of the $\mathrm{H}$-bonds in the intermolecularly $\mathrm{H}$-bonded structure, and process II is that of the orientation of the other dipolar groups, such as the -OR group. Process III is the usual JohariGoldstein process. For 5-methyl-2-hexanol, the mode-coupling and another theory by Souletie and Bertrand [J. Phys. I 1, 1627 (1991)] seem to agree with the relaxation rate of processes I and II, and predict temperatures for $10^{-4} \mathrm{~Hz}$ relaxation rate, within a few degrees of that expected. (C) 2000 American Institute of Physics. [S0021-9606(00)51705-X]
\end{abstract}

\section{INTRODUCTION}

One of the earliest studies of dielectric relaxation had shown that most of the orientation polarization in a variety of supercooled alcohols ${ }^{1-5}$ and amides ${ }^{6,7}$ decays exponentially, according to the Debye-type relaxation, and the temperature dependence of the relaxation dynamics is non-Arrhenius. Because such a behavior is contrary to the currently held view, that a non-Arrhenius relaxation dynamics of supercooled liquids corresponds to a nonexponential decay of polarization, attempts have been made recently to determine if there is an alternative explanation for such a relaxation dynamics. From a detailed study 8,9 of a supercooled primary alcohol, 1-propanol, it has been concluded that $\sim 95.5 \%$ of its total orientation polarization at $119.7 \mathrm{~K}$, which decays exponentially, is not associated with the liquid's structural relaxation, or its viscosity, and it is called process I. The remainder of the polarization is resolvable in two relaxation processes, II and III, the third being the fastest. Process II, which contributes to $\sim 4.5 \%$ of the polarization, has a distribution of relaxation times. The relaxation rate of both processes, I and II, was found to vary with the temperature in a non-Arrhenius manner. Process III was also found to have a distribution of relaxation times and its average time to vary according to the Arrhenius relaxation.

Our original understanding of the exponential decay of dielectric polarization was based on the premise that dipolar reorientation can occur only after the H-bond is broken in the intermolecularly $\mathrm{H}$-bonded structure. As the H-bond break-

\footnotetext{
a) Author to whom correspondence should be addressed. Electronic mail: jvij@tcd.ie
}

ing and reforming follows a first-order rate kinetics, the decay of the polarization is expected to be of first-order, or exponential. This view has now been discussed in terms of the rotational blocking for a certain period, referred to as the residence time in the intermolecularly $\mathrm{H}$-bonded state of the liquid. ${ }^{10}$ On the premise that the prominent Debye-type relaxation in 1-propanol is irrelevant to structural relaxation and therefore to viscosity, process II was seen as equivalent to the $\alpha$-relaxation process in a molecular liquid. ${ }^{8,9}$ The magnitude of this asymmetrically broadened process decreased with increase in the temperature, as expected from the Curie law, and its relaxation rate varied with temperature in a nonArrhenius manner.

The new conclusion ${ }^{8,9}$ is significant because the contribution to permittivity, $\Delta \epsilon$, from molecular motions involved in the structural relaxation is $\sim 2.5$, or $\sim 4.5 \%$ of the static permittivity, $\epsilon_{s}$ of 1-propanol, which means that the number of dipoles contributing to the structural relaxation in 1-propanol is much smaller than the total number present in the liquid. For that reason, it seemed necessary to investigate the relaxation dynamics of other supercooled viscous liquids which may show a Debye-type relaxation with a nonArrhenius relaxation dynamics. Here we describe the study of a secondary alcohol, 5-methyl-2-hexanol, which shows a Debye-type relaxation like that of 1-propanol. A preliminary study of the Johari-Goldstein ${ }^{11,12}$ relaxation in 5-methyl-2hexanol was reported earlier. ${ }^{13}$

\section{EXPERIMENTAL METHODS}

The dielectric cell used was a miniature, tunable parallel plate condenser containing 18 plates, with an air capacitance 


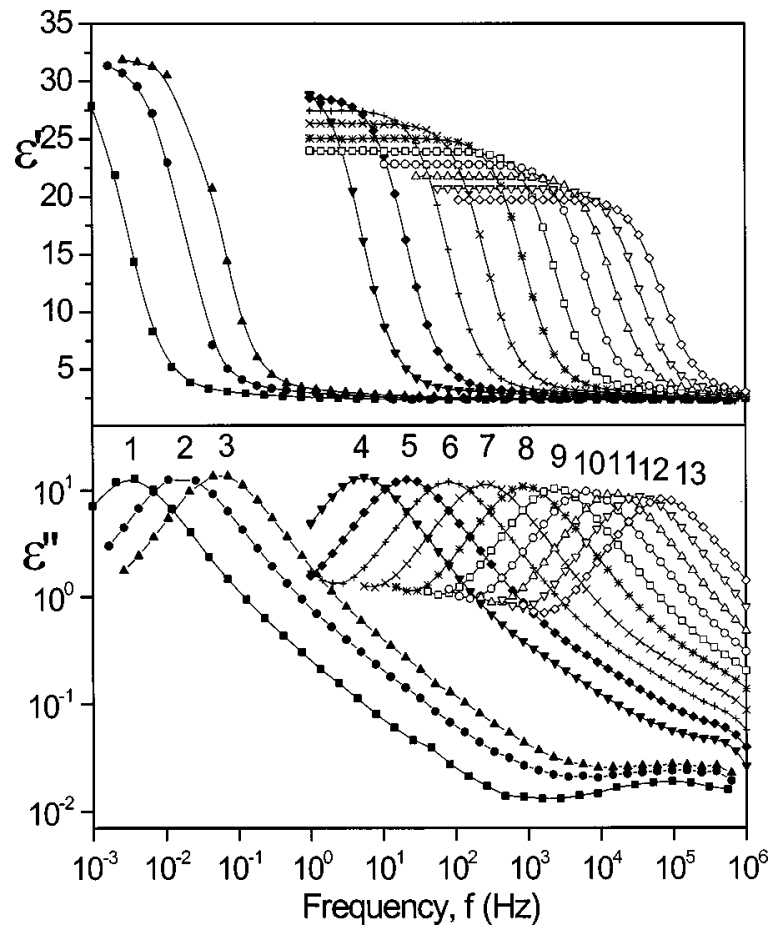

FIG. 1. The $\epsilon^{\prime}$ and $\epsilon^{\prime \prime}$ spectra of 5-methyl-2-hexanol at several temperatures: 155.4 K, 158.2 K, 160.9 K, 172.6 K, 177.3 K, 184.4 K, 187.3 K, 193.1 K, $198 \mathrm{~K}, 203.3 \mathrm{~K}, 208.5 \mathrm{~K}, 213.6 \mathrm{~K}, 218.6 \mathrm{~K}$.

of nominally $16 \mathrm{pF}$. (This conveniently available capacitor seems superior to the parallel plates separated by spacers used successfully up to $1 \mathrm{MHz}$ frequency range by several groups.) The cell and the sample's temperature was controlled by keeping it inside a cryostat, model special Spectrodn 20 Spectrostat, purchased from Oxford Instruments. The temperatures were not programmed. Rather, these were set and controlled isothermally at the desired value by its manual setting. The temperature was controlled to within $\pm 0.1 \mathrm{~K}$ over a period of up to $8 \mathrm{~h}$ required for measurements at the lowest temperature in the lowest frequency range. The dielectric permittivity $\epsilon^{\prime}$ and loss $\epsilon^{\prime \prime}$ were measured over the frequency range, $1 \mathrm{mHz}$ to $1 \mathrm{MHz}$ using a Solartron FRA$1255 \mathrm{~A}$ frequency response analyzer interfaced with a Chelsea dielectric interface. 5-methyl-2-hexanol was of purum grade purchased from Fluka AG. Switzerland, and used as such.

\section{RESULTS}

The dielectric permittivity $\epsilon^{\prime}$, and loss $\epsilon^{\prime \prime}$, spectra of 5-methyl-2-hexanol (abbreviated as 5M2H henceforth) at selected temperatures is shown in Fig. 1. A typical spectra measured at $158.2 \mathrm{~K}$ is shown in Fig. 2. It was analyzed using the WINDETA program provided by Novocontrol. This computational algorithm has been used generally in the analysis of the dielectric data by Fischer and co-workers (see, for example, Ref. 8). The equation used is given by Havriliak-Negami, ${ }^{14}$ together with an ionic conductivity term

$$
\epsilon^{*}(\omega)=\epsilon_{\infty}+\sum_{i=1}^{i=n} \frac{\epsilon_{s, i}-\epsilon_{\infty, i}}{\left[1+\left(i \omega \tau_{\mathrm{HN} i}\right)^{\alpha_{i}}\right]^{\beta_{i}}}-\frac{j \sigma}{\epsilon_{0} \omega^{s}},
$$

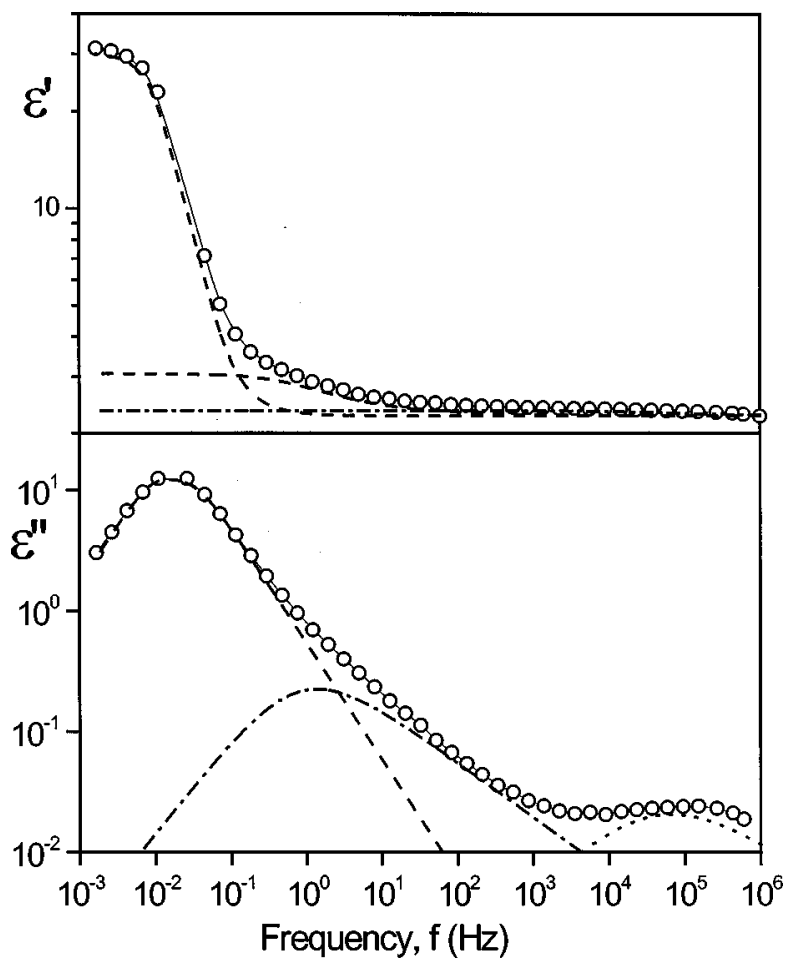

FIG. 2. The resolution of the three relaxation processes in 5-methyl-2hexanol at $158.2 \mathrm{~K}$. The parameters used for the calculations are $\Delta \epsilon_{1}$ $=28.07, \alpha_{1}=0.987, \beta_{1}=0.961, \tau_{\mathrm{HN} 1}=9.86 \mathrm{~s} ; \Delta \epsilon_{2}=0.803, \alpha_{2}=0.574, \beta_{2}$ $=0.794, \quad \tau_{\mathrm{HN} 2}=0.22 \mathrm{~s} ; \quad \Delta \epsilon_{3}=0.087, \quad \alpha_{3}=0.624, \quad \beta_{3}=0.737, \quad \tau_{\mathrm{HN} 3}=3.7$ $\times 10^{-6} \mathrm{~s}$.

where the empirical parameters $\alpha_{i}$ and $\beta_{i}$ determine the symmetric and asymmetric distributions ${ }^{14}$ of relaxation times of the spectra, $\epsilon_{s, i}$ and $\epsilon_{\infty, i}$ the limiting low- and highfrequency values of the permittivity of the individual relaxation spectra, and $\tau_{\mathrm{HNi}}$ its characteristic, or in this case called Havriliak-Negami (HN), relaxation time. $\sigma$ is the specific ionic conductivity, $\epsilon_{0}$ is the permittivity of free space, $s$ is a parameter (describing the frequency dependence of the ionic conductivity, usually the mobility of ions). $n$ is the number of relaxation processes. This is taken in this case to be unity. In such a case $\sigma$ becomes the dc conductivity. The frequency of the relaxation peak was calculated from ${ }^{8}$

$f_{m, i}=\left(2 \pi \tau_{\mathrm{HN} i}\right)^{-1}\left[\sin \frac{\alpha_{i} \pi}{2+2 \beta_{i}}\right]^{1 / \alpha_{i}}\left[\sin \frac{\alpha_{i} \beta_{i} \pi}{2+2 \beta_{i}}\right]^{-1 / \alpha_{i}}$,

frequency $f_{m, 1}$ is the frequency of maximum dielectric loss for the first and the slowest relaxation. Process I is plotted logarithmically against the reciprocal temperature in Fig. 3, where the dc conductivity $\sigma$ divided by $f_{m, 1}$ and multiplied by an arbitrary factor has also been plotted. This plot in particular shows that the rate of decrease of dc conductivity with a decrease in temperature is lower than a corresponding decrease in $f_{m, 1}$. This in particular shows that the mechanism of the relaxation process is different than for that of the dc conductivity, the latter being entirely translational. The magnitude of $\Delta \epsilon_{i}$ for each of the three relaxations is plotted logarithmically against the temperature in Fig. 4. The continuous line in Fig. 2 is the curve calculated with the values of the parameters obtained from the best fits. 


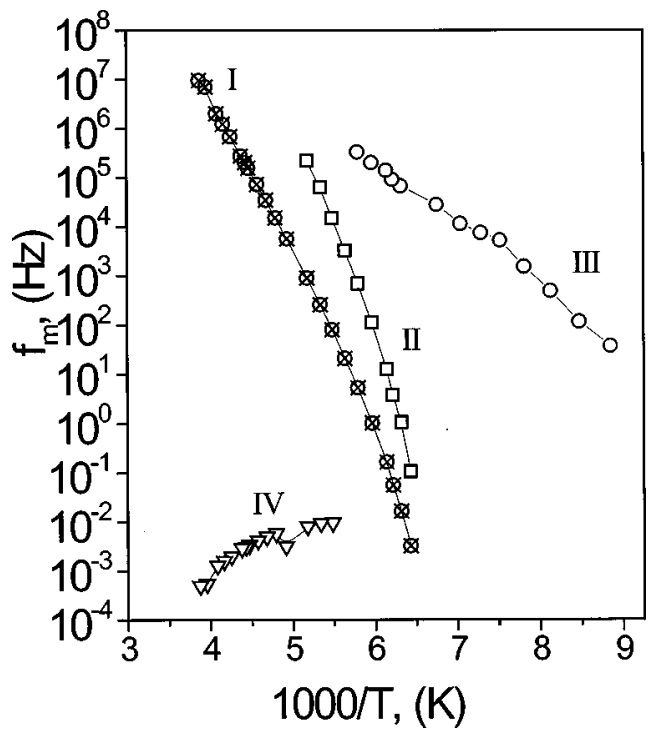

FIG. 3. The plots of $\log f_{m}$ for the $\alpha$-relaxation and the Johari-Goldstein relaxation processes against the reciprocal temperature for 5-methyl-2hexanol (I- $f_{m, 1}$, II- $f_{m, 2}$, III- $\left.f_{m, 3}\right)$. Also plotted logarithmically is the product of the dc conductivity and $1 / f_{m, 1}$ multiplied by an arbitrary factor (IV).

\section{DISCUSSION}

The occurrence of three relaxation process I, II, and III observed here is similar to that observed by Hansen et al. ${ }^{8}$ for 1-propanol. But this observation for supercooled alcohols as a class of liquids is not new, ${ }^{1-4}$ nor is the observation that the first two relaxations have a non-Arrhenius $T$-dependence of their relaxation times. Both features have been clearly

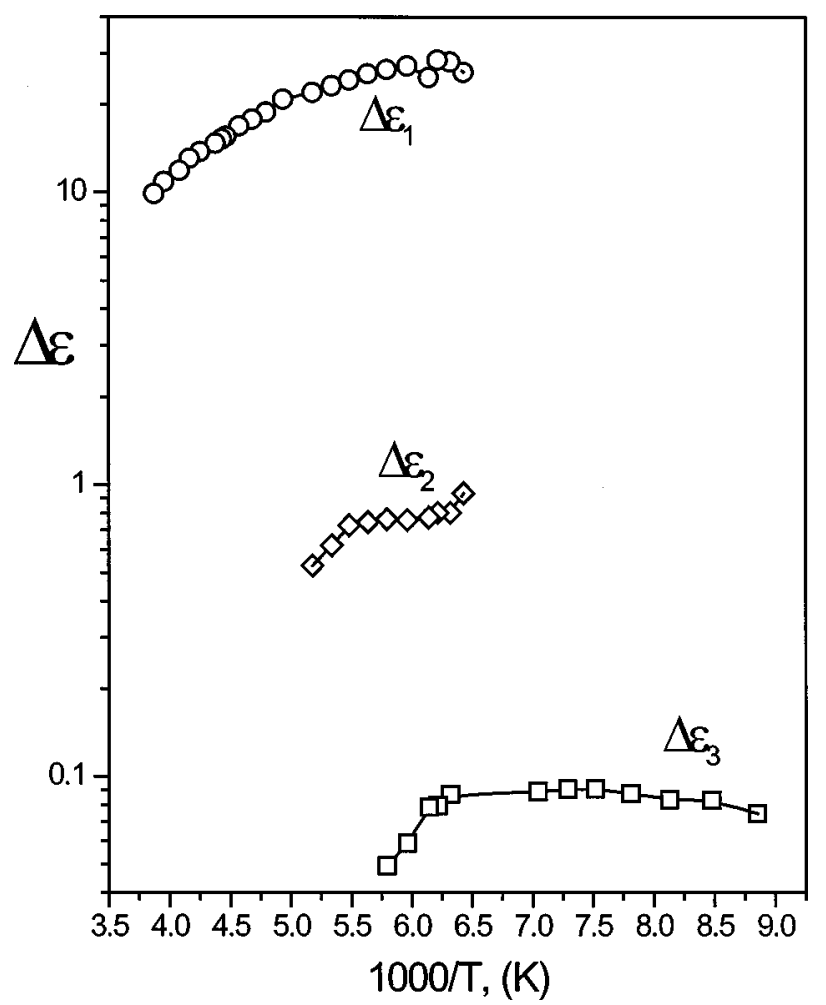

FIG. 4. The plots of the magnitude of relaxation for 5-methyl-2-hexanol against the reciprocal temperature. observed for 99\% ethanol-water and 95\% ethanol-water mixture (See Fig. 6 in Ref. 2), although somewhat less clearly in methanol, ${ }^{4}$ butanols, ${ }^{2}$ and octanols. ${ }^{5}$ As seen in Fig. 2, process I has the Debye-type characteristics, and the faster processes; II and III have the characteristics of a distribution of relaxation times. Nearly $96 \%$ of the polarization relaxes by process I and the remainder of $\sim 4 \%$ by processes II and III. This magnitude is also similar to that observed for 1-propanol. Therefore, at first sight it seems that the interpretation of the dielectric behaviors of the two alcohols, one primary and the other secondary, may be similar. But a detailed consideration shows that this may not be the case, as follows.

Hansel et al. ${ }^{8}$ have suggested that process $\mathrm{I}$ in 1-propanol involves H-bonds, but the dipolar motion involving these bonds occurs in manner that does not contribute to the viscosity or structural relaxation of 1-propanol. It seems to us that the only manner in which that can occur is by proton transfer through the $\mathrm{H}$-bonds in an immobile structure, and if that were the case, the relaxation time in alcohols would be insensitive to pressure. ${ }^{15}$ But the pressuresensitivity of the relaxation in supercooled alcohols in general has been found to be very high, as is indicated by the large volume of activation determined by Gilchrist et al. ${ }^{16}$ Scaife, ${ }^{17}$ Scaife, ${ }^{18}$ Johari and Dannhauser, ${ }^{19}$ and Vij et al. ${ }^{20}$ If it was through a mechanism of displacement of charges ${ }^{21}$ through the structure then it would lead to a distribution of relaxation times, contrary to observations. It is also not known whether making and breaking of hydrogen bonds alone leads to a fluctuation in the dipole moment.

Moreover, the high value of $\epsilon_{s}$ of most alcohols has been explained ${ }^{1-4,22-26}$ in terms of the orientational correlation factor $g$ as given by Kirkwood, ${ }^{27}$ and incorporated in the Onsager-Kirkwood-Fröhlich equation. ${ }^{28}$ When its value is greater than unity, it indicates a parallel dipolar correlation, which occurs when the $\mathrm{ROH}$ molecules associate intermolecularly by $\mathrm{H}$-bonds to form linear chain structures. This is a generally accepted molecular interpretation for the unexpectedly larger values of $\epsilon_{s}$ observed for $\mathrm{H}_{2} \mathrm{O},{ }^{29}$ amides ${ }^{4,5}$ and alcohols, both primary and secondary. ${ }^{1-4,22-26}$ The mechanism of the relaxation would thus be associated with the breaking and reforming of the H-bonded structure and the reorientation of the dipoles, and there seems to be no obvious manner in which a dipolar reorientation would not contribute to the viscosity and configurational changes in the liquid. Indeed, the relatively large magnitude of the differential scanning calorimetry (DSC) endotherm at the glass transition region of alcohols, including 5-methyl 2-hexanol $(5 \mathrm{M} 2 \mathrm{H})$, has shown that configurational relaxation involves more than $4 \%$ of the molecules, a percentage associated with process II, which alone is seen to be associated with viscosity and structural relaxation of 1-propanal.

It is likely that the mechanism for process I contributes to the structural relaxation and viscosity, as seen for other alcohols. ${ }^{30}$ With that similarity in mind, we propose that the molecular mechanism for process I is as given by Johari and Dannhauser. ${ }^{19}$ The intermolecular H-bond in a liquid alcohol breaks and reforms, which is equivalent to the structural fluctuations. Once an H-bond is broken, the surroundings of the 
dipole relax faster than its own orientation, so that the surroundings of all molecules are the same on a time average, and a single set of activation parameters would be sufficient to define the dipolar reorientation. This leads formally to the Debye-type relaxation. As the energy of the dipolar reorientation depends upon the interaction of the reorienting dipole and its surroundings, the activation energy would be influenced by the H-bond energy, but will not be equated to it. It would become temperature-dependent and pressuredependent in as much as the changes in the temperature and pressure alter the reorienting molecule's interaction energy with its surrounding molecules through a change in the average volume. Since the equilibrium polarization involves a positive correlation between the near-neighbor dipoles, the dipole reorientation may not occur by a series of steps, but by a concerted motion of the reference dipole and its neighbors, similar to that in a cooperative phenomena.

Process II has been interpreted as the $\alpha$-relaxation, which is normally observed in supercooled liquids and attributed to translational-rotational molecular motions whose freezing out on the time scale of one's experiment causes the liquid's vitrification. ${ }^{8}$ In this sense, 1-propanol showed the dielectric relaxation behavior of a typical low-molecular weight supercooled liquid, if the molecular dynamics of process I, presumably associated with H-bonds, is ignored. It seems unlikely that process II in $5 \mathrm{M} 2 \mathrm{H}$ may be interpreted in the same manner as for 1-propanol, ${ }^{8}$ i.e., in terms of the reorientation of the free molecules, i.e., molecules which are not intermolecularly $\mathrm{H}$-bonded to their neighbors. The reason is that when the temperature is decreased, intermolecular $\mathrm{H}$-bond association increases and the number of free molecules decreases. Therefore, one expects the magnitude of the relaxation to decrease, not to increase. As seen in Fig. 4, this magnitude instead increases on decreasing the temperature.

Therefore, we propose that the mechanism for process II is similar to that proposed by Dannhauser, ${ }^{4}$ i.e., that this process, "intermediate dispersion" in his terminology, originates in the H-bonded linear chain structure, and that -OR group rotation, as proposed by Hassion and Cole,$^{31}$ may be one such possibility. This is consistent with the observation that its magnitude increases on decreasing the temperature. We conclude that process II in $5 \mathrm{M} 2 \mathrm{H}$ may not be attributable to the orientation of the free H-bonded molecules. ${ }^{8}$ Its connection with the three processes observed in the study of Garg and Smyth $^{32}$ seems also unjustified in that their study was carried out at 193-313 K range in the microwave frequency range, and at that temperature and frequencies process II and III in 1-propanol as well as $5 \mathrm{M} 2 \mathrm{H}$ would be
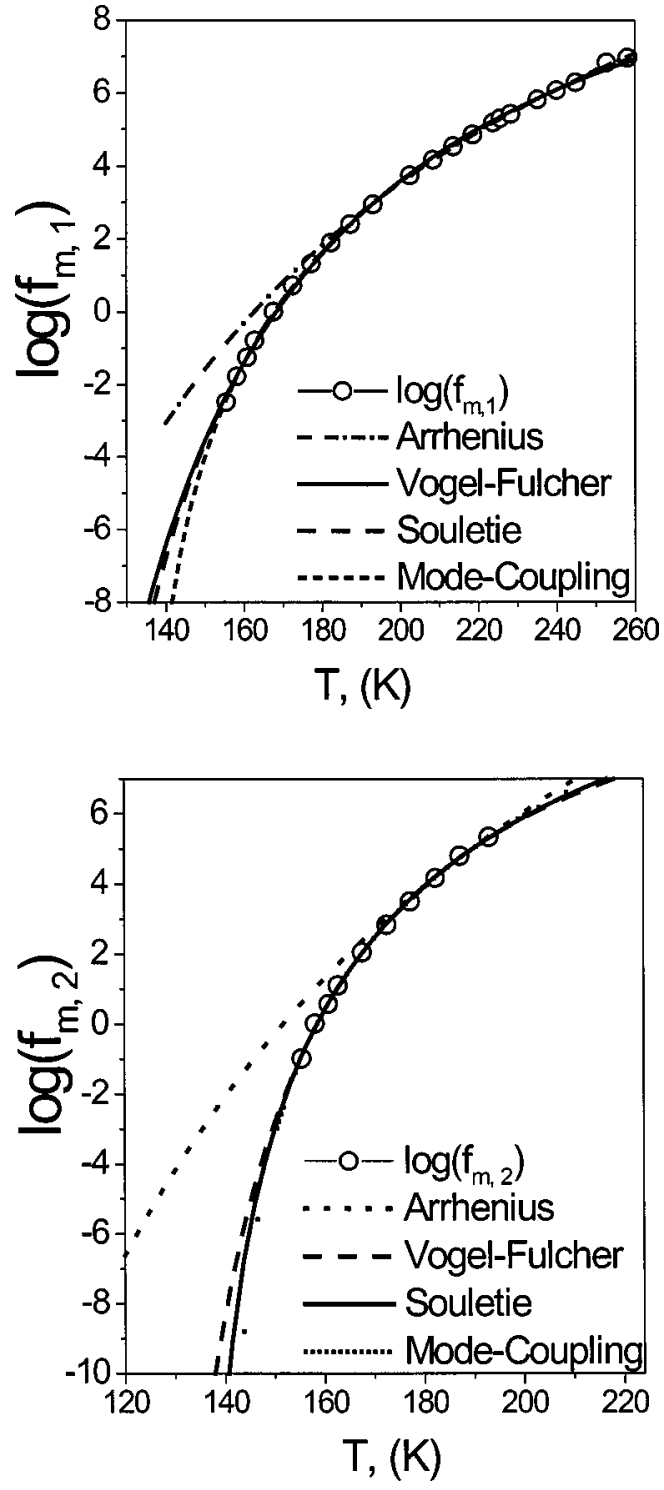

FIG. 5. The fitting of the various equations to the temperature dependence of the relaxation rate of processes I (top panel) and II (bottom panel). The equations and the parameters are given in Table I.

merged to become one, leaving only two, or possibly only one process at high temperatures.

Process III is seen as the usual Johari-Goldstein relaxation, ${ }^{8,9,12,13,33}$ which corresponds to localized motions of molecules, and which occurs both in the liquid and the vitrified states. There is little doubt that its characteristics agree with those observed for $5 \mathrm{M} 2 \mathrm{H}$.

Because the mode coupling, ${ }^{34}$ and another theory by

TABLE I. The values of parameters obtained by fitting the four equations to the relaxation rate data of 5-methyl-2-hexanol. Values before the brackets in the last three columns are for process I, and those within the brackets for process II, temperature in Kelvin.

\begin{tabular}{lcccc}
\hline \hline & Function & $A$ & $B, \gamma$ & $T_{0}, T_{c}$ \\
\hline Arrhenius & $\log f=A-B / T$ & $18.95(25)$ & $3081(3799)$ & 0 \\
Vogel-Fulcher & $\log f=A-B /\left(T-T_{0}\right)$ & $13.63(12.4)$ & $1205(575.5)$ & $79.8(112.25)$ \\
Mode-Coupling & $\log f=A+\gamma \cdot \log \left[\left(T-T_{c}\right) / T_{c}\right]$ & $7.3(10.2)$ & $12.33(11.36)$ & $133.9(140.75)$ \\
Souletie & $\log f=A+\gamma \cdot \log \left[\left(T-T_{c}\right) / T\right]$ & $14.24(14.3)$ & $29.59(17.2)$ & $112.7(135.22)$ \\
\hline \hline
\end{tabular}


Soultie and Bertrand ${ }^{35}$ predict a different temperature dependence of the relaxation rate than predicted by the Arrhenius or the Vogel-Fulcher-Tamman ${ }^{36-38}$ equations, there is an interest in fitting the equations from these theories to the $\ln \left(f_{m}\right)$ data, as done by Stickel et al. ${ }^{39}$ This comparative fitting is shown in Fig. 5 for both process I and II, and the parameters for the fit are listed in Table I. The plots in Fig. 5 show that there is little distinction between the data calculated from the mode coupling, ${ }^{34}$ Souletie and Bertrand, ${ }^{35}$ and Vogel-Fulcher-Tamman ${ }^{36-38}$ equations at $10^{-1} \mathrm{~Hz}<f_{m}$ $<10^{6} \mathrm{~Hz}$. The differences are much more prominent at low temperatures, and there is no more than $2-4 \mathrm{~K}$ difference (Fig. 5) between the prediction of the glass transition temperature (at $f_{m}=10^{-4} \mathrm{~Hz}$ ) by the three equations. This observation is significant in view of the earlier observations ${ }^{9,39}$ that the two theories yield a much lower value of the glass-transition temperature than the Vogel-FulcherTamman $^{36-38}$ equation.

\section{CONCLUSION}

The dielectric relaxation dynamics in a secondary alcohol, 5-methyl-2-hexanol, involves three relaxation processes, the most prominent of which is of the Debye-type, the other two have an asymmetric distribution of relaxation times. On cooling, the first two processes appear to merge in the vicinity of $140 \mathrm{~K}$. On heating, the fastest process merges with the intermediate process at $\sim 200 \mathrm{~K}$. It is proposed that the mechanism of the first relaxation processes is the breaking of the H-bond and reorientation of the dipole, and that of the second, the reorientation of the -OR groups in the H-bonded structure. The equations from both the mode-coupling ${ }^{34}$ and Souletie and Bertrand theories of relaxation yield values of the relaxation rate closely similar to those obtained from the Vogel-Fulcher-Tamman ${ }^{36-38}$ equation, and little distinction is found between the values calculated at temperatures close to the glass-transition temperature.

\section{ACKNOWLEDGMENTS}

We thank the American Petroleum Research Fund administered by the American Chemical Society, PRF \#33221AC7, for funding this work. We are extremely grateful to Professor G. P. Johari for having suggested the work, for his supervision, and for the very many stimulating discussions during the course of writing of this article.
${ }^{1}$ R. H. Cole and D. W. Davidson, J. Chem. Phys. 20, 1389 (1952).

${ }^{2}$ F. X. Hassion and R. H. Cole, J. Chem. Phys. 23, 1756 (1955).

${ }^{3}$ W. Dannhauser and R. H. Cole, J. Chem. Phys. 23, 1762 (1955).

${ }^{4}$ D. J. Denney and R. H. Cole, J. Chem. Phys. 23, 1767 (1955).

${ }^{5}$ W. Dannhauser, J. Chem. Phys. 48, 1918 (1968).

${ }^{6}$ S. J. Bass, W. I. Nathan, R. M. Meighan, and R. H. Cole, J. Phys. Chem. 68, 509 (1964).

${ }^{7}$ W. Dannhauser and G. P. Johari, Can. J. Chem. 46, 3143 (1968).

${ }^{8}$ C. Hansen, F. Stickel, T. Berger, R. Richert, and E. W. Fischer, J. Chem. Phys. 107, 1086 (1997).

${ }^{9}$ A. Kudlik, C. Tschirwitz, S. Benkhof, T. Blochowicz, and E. Rössler, Europhys. Lett. 40, 649 (1997); J. Mol. Struct. (G. P. Johari special issue) 479, 201 (1999).

${ }^{10}$ H.-G. Kohne and M. Stockhausen, Z. Naturforsch. A 50, 352 (1995).

${ }^{11}$ G. P. Johari and M. Goldstein, J. Chem. Phys. 53, 2372 (1970).

${ }^{12}$ G. P. Johari, J. Chem. Phys. 58, 1766 (1973).

${ }^{13}$ J. K. Vij, J. Mol. Liq. (B. K. P. Scaife special issue) 69, 19 (1996).

${ }^{14}$ S. Havriliak, Jr. and S. Negami, Polymer 8, 161 (1967); J. Polym. Sci., Part C: Polym. Symp. 14, 89 (1966).

${ }^{15}$ J. Middlehoek and C. J. F. Böttcher, Molecular Relaxation Process (Chemical Society and Academic Press, London, 1966), Chem. Soc. Spec. Publ. 20, 69 (1966).

${ }^{16}$ A. Gilchrist, J. E. R. Earley, and R. H. Cole, J. Chem. Phys. 26, 196 (1957).

${ }^{17}$ B. K. P. Scaife, Proc. Phys. Soc. London, Sect. B 68, 790 (1955).

${ }^{18}$ W. G. Scaife, Natl. Acad. Sci.-Natl. Res. Council Publ. Washington 1578, 70 (1968).

${ }^{19}$ G. P. Johari and W. Dannhauser, J. Chem. Phys. 50, 1862 (1969).

${ }^{20}$ J. K. Vij, W. G. Scaife, and J. H. Calderwood, J. Phys. D: Appl. Phys. 14, 733 (1981).

${ }^{21}$ R. Fuchs and A. von-Hippel, J. Chem. Phys. 34, 2165 (1961).

${ }^{22}$ M. Moriamez, L. Raczy, E. Constant, and A. Lebrun, J. Chim. Phys. 61, 146 (1964).

${ }^{23}$ G. P. Johari and W. Dannhauser, J. Chem. Phys. 48, 5114 (1968).

${ }^{24}$ T. Chen, W. Dannhauser, and G. P. Johari, J. Chem. Phys. 50, 2046 (1969).

${ }^{25}$ R. G. Bennett, G. H. Hall, and J. H. Calderwood, J. Phys. D: Appl. Phys. 6, 781 (1973).

${ }^{26}$ J. K. Vij, W. G. Scaife, and J. H. Calderwood, J. Phys. D: Appl. Phys. 11, 545 (1978).

${ }^{27}$ J. G. Kirkwood, J. Chem. Phys. 4, 592 (1936); J. Chem. Phys. 7, 911 (1939).

${ }^{28}$ See for a detailed analysis, B. K. P. Scaife, Principles of Dielectrics (Clarendon, Oxford, 1998), p. 200.

${ }^{29}$ See for a detailed discussion, J. B. Hasted, Aqueous Dielectrics (Chapman and Hall, London, 1973).

${ }^{30}$ G. P. Johari and W. Dannhauser, J. Chem. Phys. 51, 1626 (1969).

${ }^{31}$ F. X. Hassion and R. H. Cole, Nature (London) 172, 212 (1953).

${ }^{32}$ S. K. Garg and C. P. Smyth, J. Phys. Chem. 69, 1294 (1965).

${ }^{33}$ O. E. Kalinovskaya and J. K. Vij, J. Chem. Phys. 111, 10979 (1999).

${ }^{34}$ W. Götze and L. Sjögren, Rep. Prog. Phys. 55, 241 (1992).

${ }^{35}$ J. Souletie and D. Bertrand, J. Phys. I 1, 1627 (1991).

${ }^{36}$ H. Vogel, Phys. Z. 22, 645 (1921).

${ }^{37}$ G. S. Fulcher, J. Am. Ceram. Soc. 8, 339 (1923).

${ }^{38}$ G. Tamman and W. Hesse, Z. Anorg. Allg. Chem. 156, 245 (1926).

${ }^{39}$ F. Stickel, E. W. Fischer, and R. Richert, J. Chem. Phys. 104, 2043 (1996). 